\title{
Sustained proton pump inhibitor
} deprescribing among dyspeptic patients in general practice: a return to selfmanagement through a programme of education and alginate rescue therapy. A prospective interventional study.

Cathal Coyle, PhD ${ }^{1 *}$, Russell Symonds ${ }^{2}$, Jane Allan, RGN ${ }^{3}$, Sarah Dawson, RGN ${ }^{4}$, Sheldon Russell, BSc (Hons), MRPharmS IPresc ${ }^{5}$, Adam Smith, PhD ${ }^{6}$, Colin Daff, BSc, DMS 7 , Helen Kotze, PhD ${ }^{8}$

${ }^{1}$ Global Medical Affairs Lead, Global Medical Affairs, Reckitt Benckiser, Slough, Berkshire, UK; ${ }^{2} \mathrm{NHS}$ Business Manager, NHS team, Reckitt Benckiser, Slough, UK; ${ }^{3}$ Nurse Advisor, Ashfield: Patient Solutions, Ashfield Healthcare, Leicester, UK; ${ }^{4}$ Nurse Advisor, Ashfield: Patient Solutions, Ashfield Healthcare, Leicester, UK; ${ }^{5}$ Practice Support Pharmacist, Medicines Management, Barnet Clinical Commissioning Group, London, UK; ${ }^{6}$ Evidence and Outcomes Manager, Health Outcomes, Evidence Generation \& Clinical Research, Reckitt Benckiser, Slough, UK; ${ }^{7}$ Assistant Director, Medicines Management, Barnet Clinical Commissioning Group, London, UK; ${ }^{8} \mathrm{Global}$ Medical Affairs Manager, Global Medical Affairs, Reckitt Benckiser, Slough, UK
*For correspondence: Cathal. Coyle@rb.com

Competing interest: See page 8

Received: 21 March 2019

Accepted: 10 May 2019

Published: 10 July 2019

(This article is Open Access: CC BY license (https://creativecommons.org/licenses/by/4.0/)

Author Keywords: alginates, proton pump inhibitor, dyspepsia, primary health care, self-care

Copyright (C) 2019, The Authors; DOI:10.3399/

bjgpopen19X101651

\section{Abstract}

Background: Dyspepsia guidelines recommend that patients treated with proton pump inhibitors (PPIs) should step down to the lowest effective dose or return to self-care, but rebound hyperacidity can make this difficult. Many patients continue on PPIs in the long term, which may lead to safety and financial implications.

Aim: To determine if a nurse-led educational support programme and rescue therapy for rebound symptoms can help patients achieve a sustained reduction in PPI use.

Design \& setting: A prospective interventional study was conducted at 26 surgeries across the UK.

Method: Adult patients, treated with PPIs for $\geq 2$ consecutive months with an active repeat prescription, were invited to a 20-minute dyspepsia clinic appointment with a trained nurse adviser. An action plan to reduce and/or stop their PPI usage was agreed and alginate supplied for the self-management of rebound symptoms. After 12 months, PPI status was reviewed and prescribing cost savings calculated.

Results: After 12 months, $75.1 \%$ of 6249 eligible patients stepped down or off PPIs (35.3\% stepped off; $5.0 \%$ stepped down then off; $34.8 \%$ stepped down only), while $8.7 \%$ of patients had reverted to their original PPI dose. PPI prescriptions fell from 89915 to 45880 and alginate prescriptions increased from 2405 to 6670 . An average of 1.7 bottles (500 ml each) of alginate were used per patient who stepped down or off. Estimated annual cost-saving on prescriptions was $£ 31716.30$.

Conclusion: A programme of education and short-term rebound symptom management helped the majority of patients to successfully step down or off PPIs, significantly reducing the potential risks associated with chronic therapy. 


\section{How this fits in}

For many patients with dyspepsia on long-term PPI treatment, the potential for harm may be greater than the therapeutic advantage. However, symptoms related to rebound acid hypersecretion may represent a barrier to successfully reducing or stopping PPI treatment. A nurse-led programme of education and alginate prescribing for short-term rebound symptom management achieved sustained reductions in PPI use in 26 surgeries across the UK. This relatively simple intervention may significantly impact the risk of long-term harm to PPI-treated patients and empower them to take a more active role in their own care.

\section{Introduction}

Since their clinical introduction during the 1980s, PPIs have become one of the most frequently prescribed drugs worldwide, with especially high rates of use in some European countries. ${ }^{1}$ As potent inhibitors of gastric acid secretion, they have revolutionised the treatment of acid-related conditions, including peptic ulceration ${ }^{2}$ and oesophagitis related to gastro-oesophageal reflux disease (GORD). ${ }^{3}$

While the short-term risk-benefit profile for PPIs is favourable, the impact of their widespread, long-term use is increasingly uncertain, ${ }^{4-7}$ with national drug agencies warning about their use long term and/or at high doses. ${ }^{8,9}$ Potential adverse effects could have a significant negative impact at a population level. ${ }^{10}$ Indeed, a recent large-scale study that followed PPI versus non-PPI users for a median of 5.71 years showed a significant association between PPI use and all-cause mortality, and the risk increased with prolonged use. ${ }^{10}$ Potential confounders associated with observational studies means results should be interpreted with caution, but the body of evidence does suggest the need to limit long-term PPI use to justified medical instances. For example, in patients with Barrett's oesophagus, high-dose PPI use has been shown to significantly improve outcomes over a 9-year period. ${ }^{11}$

The UK guidelines for the management of dyspepsia and GORD recommend that patients receiving long-term PPI treatment are regularly reviewed and encouraged to step down to the lowest effective $\mathrm{PPI}$ dose, or return to self-care with antacids and/or alginates to manage intermittent symptoms. ${ }^{12}$ Despite this, a recent study reported that $60 \%$ of long-term PPI users in the UK had not attempted to discontinue or step down PPI dose ${ }^{13}$ and Health Survey for England revealed that PPIs were the third most commonly prescribed medicine, accounting for $11 \%$ of prescribed medicines in $2016 .{ }^{14}$ Evidence suggests that patients frequently persist on PPIs unnecessarily, and that PPIs are often prescribed without a clear indication. ${ }^{15-21}$ Furthermore, PPI treatment is often continued in GORD patients, even those with breakthrough symptoms and unsatisfactory symptom control. ${ }^{22-24}$

Action is required to support deprescribing and reduce the potential safety and financial implications of long-term PPI treatment. In practice, this can be challenging as sustained hypoacidity during treatment promotes hypergastrinaemia, causing rebound acid hypersecretion when PPI therapy is withdrawn. ${ }^{25,26}$ Stopping PPI treatment has been shown to induce acid-related symptoms after 4-8 weeks of treatment, even in previously asymptomatic individuals. ${ }^{27,28}$ While guidelines recommend restricting and deprescribing PPls, they do not describe evidence-based strategies for the management of rebound hyperacidity. Thus, potential recurrence of symptoms may be a barrier to PPI reduction, especially in patients who have responded well to therapy.

An increasing body of evidence from small-scale, single-centre studies suggests that approaches using alginate as short-term rescue therapy can effectively help patients manage rebound symptoms and return to self-care. ${ }^{29-33}$ However, the effectiveness of this approach requires confirmation in a large, multi-centre study that allows for socioeconomic and cultural diversity. The aim of this study was to evaluate the efficacy and financial impact of a nurse-led programme of education and support across different regions of the UK.

\section{Method}

\section{Study design and setting}

This was a prospective interventional study conducted at 26 surgeries across nine regional primary care organisations (PCOs) in the UK (six in England, two in Scotland, one in Wales). Eligible PPI-treated patients participated in the nurse-led Dyspepsia Therapy Review and Education Programme (DTREP). In line with 


\section{SAMPLING}

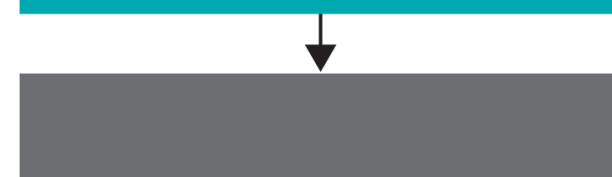

RECRUITMENT

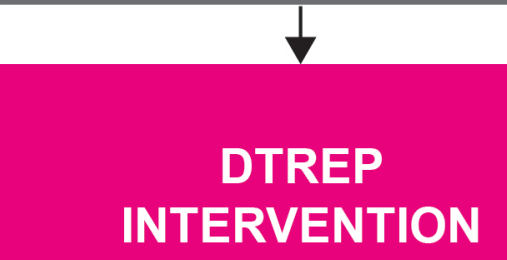

SUPPORT

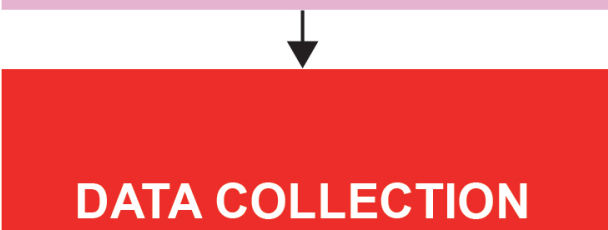

DATA ANALYSIS
Practice register and medical records screened. Inclusion and exclusion criteria applied.

Eligible patients sent written invitation to attend dyspepsia review clinic.

Patients attended a 20-minute appointment with a specialist nurse advisor. Written, informed consent obtained, dyspepsia questionnaire conducted, educational information provided, management plan agreed and alginate prescribed by practice.

Additional consultations requested

by patients if required.

Review of patients' records after 12 months.

$\mathrm{PPI}$ and alginate use recorded.

Analysis of data and any cost-savings calculated.

Figure 1 Study protocol summary

DTREP $=$ Dyspepsia Therapy Review and Education Programme. PPI = proton pump inhibitor.

best practice guidance, DTREP aims to encourage patients on long-term PPI therapy to return to self-care through a package of education and support. Informed, written consent was obtained from all patients before entering the programme. An overview of the study methodology is provided in Figure 1.

\section{Patients}

The electronic register for each surgery was searched to identify all potentially suitable patients. Eligible patients (aged 18-90 years) had an active repeat prescription for PPIs and had been treated 
with PPIs for a minimum of 2 consecutive months. Specialist nurse advisers screened medical records, applying pre-specified inclusion and exclusion criteria, before providing GPs with participant lists for approval.

Exclusion criteria included patients on Helicobacter pylori eradication therapy; with grade 3 or 4 oesophagitis, Zollinger-Ellison syndrome, terminal illness, history of oesophageal varices, strictures, or dilation; awaiting or under gastrointestinal clinic or gastroscopy review; receiving immunosuppressants, chemotherapy, or radiotherapy; or with alarm symptoms (persistent vomiting, bleeding, anaemia, unexplained weight loss, or difficulty swallowing).

Patients who were considered for step down to the lowest maintenance dose of PPI but could not proceed to self-management included those with a history of peptic ulceration associated with Campylobacter-like organism negative status, a Barrett's oesophagus diagnosis, or patients requiring continuation of non-steroidal anti-inflammatory drug (NSAID) therapy, except for those considered at high risk; that is, those with previous ulceration, those on other medication harmful to the gastric and duodenal lining, older people, and those on long-term high-dose NSAIDs (20 mg omeprazole defined as maintenance dose for NSAID coverage). Patients using aspirin or clopidogrel to prevent cardiovascular disease could be stepped off PPIs, except if considered high risk; for example, previous ulceration, taking medication harmful to the gastric/duodenal lining, and older people.

\section{Dyspepsia therapy review and education programme}

All eligible patients were sent written invitations to attend a 20-minute dyspepsia clinic appointment with a specially trained nurse adviser. During the initial visit, patients completed a patient counselling questionnaire (further information available from the authors on request) to obtain a structured history and to screen for alarm symptoms. Symptomatic patients who had not undergone screening for $H$. pylori infection may have been referred to the practice nurse for testing according to the local guidelines and were subsequently re-entered into the programme on completion of eradication therapy. As per UK National Institute for Health and Care Excellence guidelines, ${ }^{12}$ patients were given verbal and written educational information about their condition, its causes, risk factors, alternative treatment options, and lifestyle factors. This included advice about identifying personal triggers and potential dietary precipitants such as coffee, chocolate, and fatty foods. ${ }^{34}$ Risk factor management included a brief alcohol intervention and smoking cessation referral advice, if relevant. The specialist nurse adviser and the patient agreed a specific action plan to reduce and/or stop PPI usage. As part of this plan participants were prescribed, as per GP practice guidance, Gaviscon Advance, an alginate formulation ( $1 \mathrm{~g}$ sodium alginate and $200 \mathrm{mg}$ potassium bicarbonate $/ 10 \mathrm{ml}$ dose) licensed for rebound dyspepsia and breakthrough symptoms. Further appointments were offered to all patients according to their individual needs. Patients who had consented to participate but did not attend the clinic were sent a further invitation letter offering flexible appointment times. Specialist nurse advisers reviewed patients' records of PPI and alginate prescription for the 12-month period following intervention, and again at 24 months at several surgeries. Any adverse events were recorded using recognised protocols.

\section{Data collection and analysis}

All data were coded and collated in a Microsoft Excel workbook (version 6.4). Simple descriptive statistics were used to calculate the number and percentages of patients who had stepped down (reduced PPI dose) or stepped off (discontinued) PPIs after 12 months.

The net financial costs of PPI and alginate use were calculated after 12 months. All annualised cost estimates were derived from the UK Department of Health and Social Services electronic drug tariff. Annual savings were calculated by comparing PPI costs at baseline with PPI and Gaviscon Advance costs at study conclusion.

\section{Results}

\section{Patients}

Prescribing data were reviewed for 231900 patients; 14108 PPI-treated patients were identified, of whom 6249 were eligible for this study. 
Table 1 Net impact of DTREP programme on proton pump inhibitor status

\begin{tabular}{lc}
\hline Outcome & Patients, $\mathbf{n}(\%)$ \\
\hline Total eligible & $6249(100)$ \\
\hline Step down & $2173(34.8)$ \\
Step down then off & $312(5.0)$ \\
Step off only & $2206(35.3)$ \\
\hline Total successful reduction & $4691(75.1)$ \\
\hline Did not attend & $520(8.3)$ \\
Unsuccessful, reverted & $541(8.7)$ \\
Under review and/or refused & $163(2.6)$ \\
No longer active & $334(5.3)$ \\
\hline Total reduction & $1558(24.9)$ \\
\hline
\end{tabular}

DTREP = Dyspepsia Therapy Review and Education Programme.

\section{Outcomes}

\section{One-year review}

After participating in the DTREP, a total of 4691 (75.1\%) eligible patients had stepped down or off PPls after 12 months (Table 1). PPls were discontinued in $40.3 \%$ of eligible patients. Overall, around a third of patients stepped off (35.3\%), another third stepped down to a lower dose of PPI without stepping off (34.8\%), and $8.7 \%$ of patients reverted to their original PPI dose after 12 months.

In England and Wales (Figure 2), the rate of stepping off PPIs (England 43.8\%, Wales 44.6\%) was greater than stepping down (England 30.2\%, Wales $31.0 \%$, whereas data from the Scottish PCOs show greater rates of stepping down (52.6\%) versus stepping off (26.1\%). Rates of nonattendance were also noticeably higher in Scotland (14.7\%) compared with England (6.8\%) and Wales (6.7\%). No adverse events were reported during the study period.

\section{Two-year follow up}

Three of the study surgeries in England also reviewed prescribing data 24 months after implementation of DTREP. Out of 1455 PPI-treated patients, 669 were eligible. Two years after they entered DTREP, $254(38.0 \%)$ had stepped off PPIs and 174 (26.0\%) had stepped down. Nine out of the $254(3.5 \%)$ patients who stepped off had first stepped down.

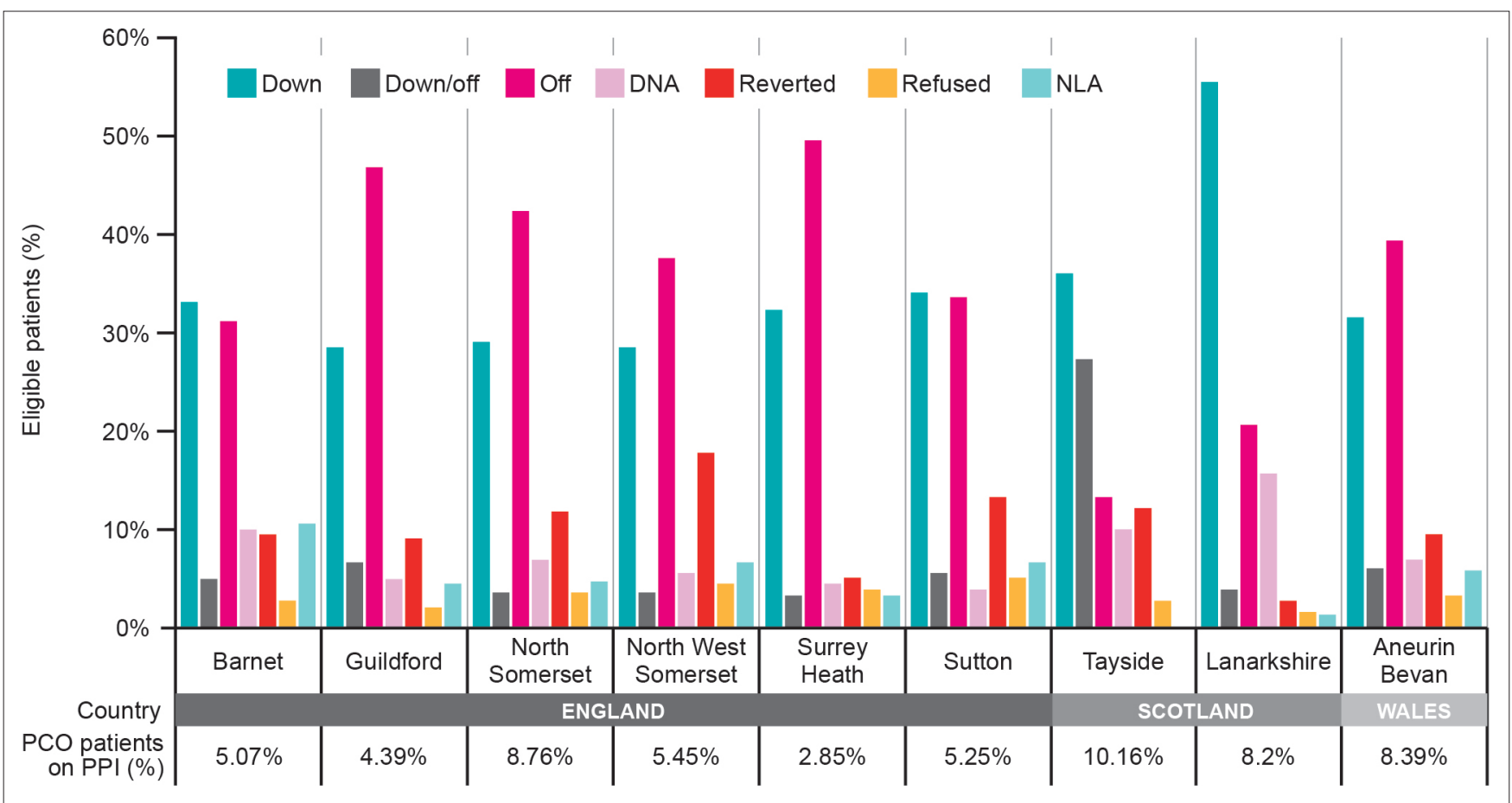

Figure 2 DTREP impact on PPI use across regional PCOs in England, Scotland, and Wales

The proportion of PPI-treated patients and change in PPI status are summarised for each study PCO.

DNA = did not attend. DTREP = Dyspepsia Therapy Review and Education Programme. NLA = no longer active. PCO = primary care organisation (clinical commissioning group). PPI = proton pump inhibitor. 


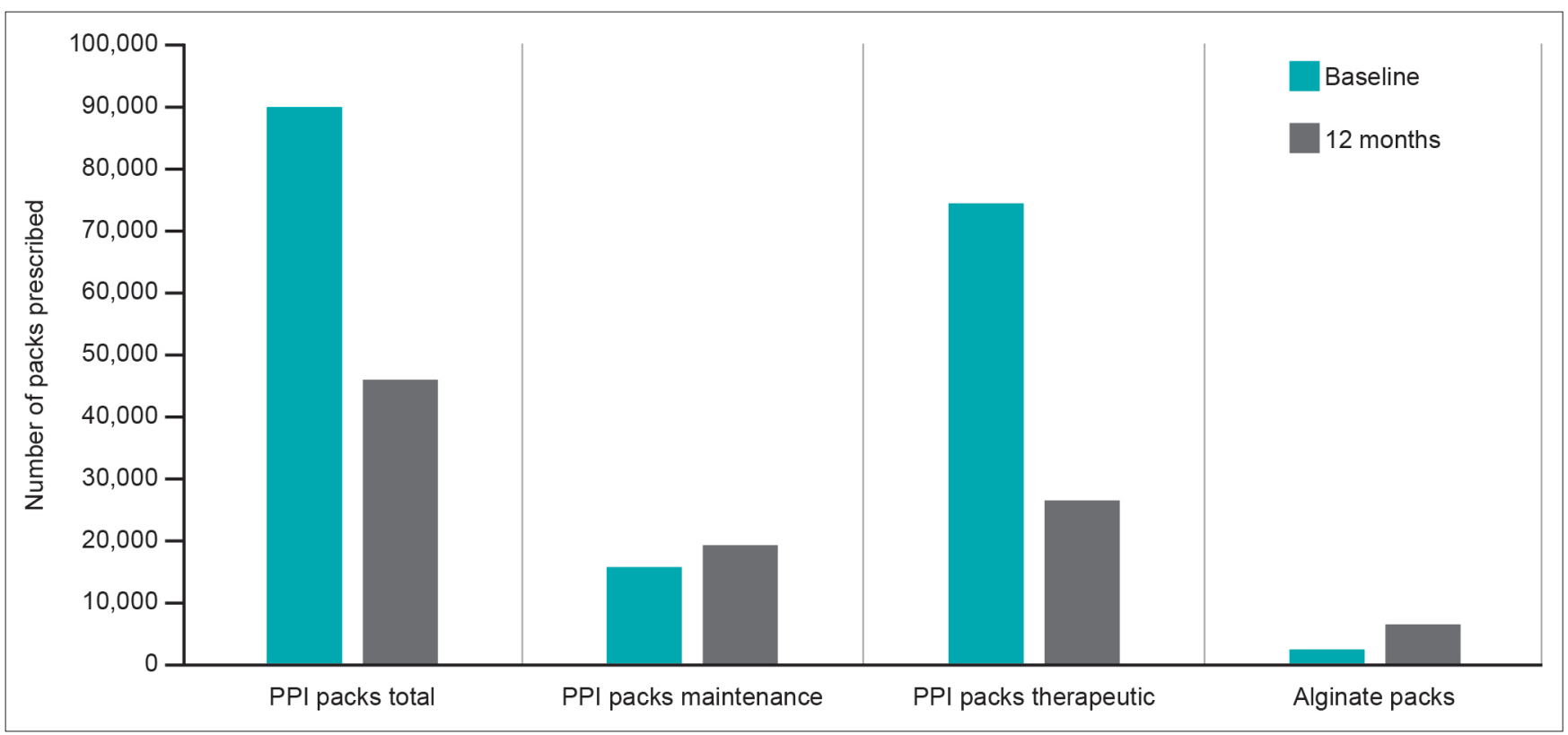

Figure 3 Net impact of DTREP programme on prescriptions

DTREP $=$ Dyspepsia Therapy Review and Education Programme. PPI = proton pump inhibitor.

After 24 months, $64.0 \%$ of patients had either stepped down or off PPIs, $14.2 \%$ were unsuccessful and/or reverted, $7.9 \%$ did not attend or were under review and/or refused to complete the programme, and $13.9 \%$ were no longer active (that is, no longer registered with the surgery).

\section{Prescriptions and costings}

Overall, there was a $49.0 \%$ reduction in PPI prescribing over the 1 -year review period (Figure 3 ), with the number of PPI prescriptions falling from 89915 to 45880 . The number of alginate prescriptions over the same period increased 2.7-fold, from 2405 to 6670. During the 12-month period, an average of 1.7 bottles ( $500 \mathrm{ml}$ each) of Gaviscon Advance were used per patient who stepped down and/or off. Taking into consideration the cost of alginate ( $f 21836.80)$, the net prescribing cost-saving from DTREP was $£ 31716.30$ per annum.

\section{Discussion}

\section{Summary}

Provision of education and support through the nurse-led DTREP was very effective at helping patients successfully reduce their PPI use across all the study PCOs. Overall, around $40 \%$ of patients stopped PPI treatment completely and 35\% reduced their dose. These changes in PPI use were achieved without any reported safety concerns and were maintained long term; PPI reduction was maintained in $75.1 \%$ and $64.0 \%$ of patients at 12 and 24 months, respectively.

An important element of DTREP was preparing patients for the possible recurrence of symptoms and provision of alginate as rescue therapy. Gaviscon Advance is a concentrated alginate formulation that forms a resilient reflux-suppressing raft at the gastro-oesophageal junction. ${ }^{35,36}$ Its rapid action makes it an ideal candidate for rescue therapy and it has been shown previously to help PPI-treated patients cope with acute breakthrough symptoms. ${ }^{37}$ Furthermore, the non-systemic, physical mode of action means it has a favourable safety profile with no drug interaction issues. ${ }^{38,39}$ During the 12-month study period, an average of 1.7 bottles of alginate were used for every patient who stepped down and/or off, suggesting that a real reduction in medication use was achieved rather than simply switching one chronic medication for another. Furthermore, implementation of DTREP was estimated to have saved more than $£ 30000$ on the annual prescribing budget across the study surgeries. 


\section{Strengths and limitations}

A notable strength of this study was the large patient sample (more than 6000 eligible PPI-treated patients), real-world setting, and regional spread of the study population, which represents diverse social, economic, and cultural groups. The overall effectiveness across the UK demonstrates the widespread feasibility of DTREP for general clinical practice. A limitation of the study was that patients were not well-characterised in terms of age, duration of PPI use, ethnic group, and health and socioeconomic status - factors that will almost certainly influence the likelihood of successful PPI reduction. The proportion of PPI-treated patients within the individual PCOs may give an indication of the general health status of the local population. The PCO with the highest proportion of PPItreated patients (Tayside, 10.2\%) had a considerably greater rate of 'step down then off' versus 'step off alone' (26.9\% versus $13.1 \%$, respectively). This contrasts with the PCO with the lowest proportion of PPI-treated patients (Surrey Heath, $2.9 \%$ ), where patients were more likely to immediately step off $(49.1 \%)$, rather than step down first (1.6\%). Rates of non-attendance were also generally higher in the $\mathrm{PCO}$ with a greater proportion of PPI-treated patients. Further investigation into how cultural, health, and socioeconomic factors influence the likelihood of step down versus step off will be important in helping to adapt DTREP to better meet the needs of the local population. Future studies should also assess the health-related quality of life benefit for patients versus the cost of the programme.

A further limitation is that, with no comparator, the relative contribution of alginate is unknown. Also, use of over-the-counter alginate or other medication for dyspepsia was not recorded, the costs of running the programme were not taken into account in the estimated overall cost-saving calculations, and there was no guarantee of consistency in approach between centres.

\section{Comparison with existing literature}

As mentioned, the relative contribution of alginate to the success of DTREP cannot be confirmed. However, previous studies using educational intervention alone had limited or no success in reducing PPI use. ${ }^{19,40,41}$ The data in this study are also consistent with previous small-scale studies involving similar interventions of educational support and alginate for rebound symptom management. ${ }^{29-33}$ One of these studies reviewed PPI use as part of a polypharmacy medicine optimisation review (PMOR). ${ }^{33,42}$ It revealed that $79 \%$ of patients using PPIs were taking four or more medications, and PMOR not only led to PPI reduction but also reduced NSAID and selective serotonin reuptake inhibitor use. This suggests that similar interventions could be applied to other areas of medicine where patients might benefit from reducing their long-term medication. Furthermore, in addition to reduced PPI prescribing, PMOR led to an unexpected decrease in gastrointestinal and endoscopy referrals, indicating a potential for cost-savings owing to reduced healthcare utilisation.

\section{Implications for practice}

PPI overprescribing has been a recognised problem for more than a decade, ${ }^{43}$ but the number of PPI items dispensed in the UK continues to rise, doubling from 29 million in 2007 to 59 million in $2017 . .^{44}$ A recent study investigating PPI prescribing in primary care found that up to $21 \%$ of PPI courses had no coded indication. ${ }^{13}$ Unnecessary PPI use puts patients at risk of side effects, such as small intestine bacterial overgrowth, ${ }^{45}$ or long-term complications, such as increased fracture risk. ${ }^{46}$ While rebound symptoms ${ }^{27}$ make it difficult for patients to reduce their PPI dose or return to self-management in line with guidelines, ${ }^{12}$ the success of DTREP emphasises that a relatively simple intervention can help patients achieve this. The results of DTREP are a reminder that high-volume prescribing can be successfully reduced, and are particularly encouraging in light of the recent focus on empowering patients with evidence-based approaches to self-care. ${ }^{47}$

A population modelling tool used DTREP data to forecast outcomes at a population level, taking into account prescribing costs and drug-related complications based on national averages. The tool shows that for 100000 registered patients, 6.1\% of whom are taking PPls, 937 patients could be stepped down or off, with a net cost-saving of $£ 13676.71$ (based on costings as of 1 August 2018).

Three out of four patients who entered the DTREP achieved a sustained reduction or complete discontinuation of PPls using alginate as a short-term rescue therapy.

Greater guidance on rebound symptom management and broader use of DTREP has the potential to significantly impact the risk of long-term harm to PPI-treated individuals. Such interventions of 
education and support will help drive implementation of evidence-based guidance and empower patients to take a more active role in their own health care.

\section{Funding}

This study was commissioned and funded by Reckitt Benckiser (RB). Writing support was provided by Lisa O'Rourke PhD at Cello Health Cypher and was funded by RB.

\section{Ethical approval}

Ethical approval was not required as the DTREP programme was an implementation of NICE guidelines and any change in medication was made by the GP. Before consenting to take part in the study, participants were given information about the study and how their data would be used.

\section{Provenance}

Freely submitted; externally peer reviewed

\section{Acknowledgements}

The authors would like to thank Mark Bradshaw of RB for development of the cost-modelling tool, Mark Spencer for his work on the pilot study at the Fleetwood Practice, Lanarkshire, and Kumar Basu for his critical review of the manuscript.

\section{Competing interests}

No authors received any financial support from RB during the conduct of the study. CC, RS, AS and HK are employees of RB.

\section{References}

1. Lanas A. We are using too many PPls, and we need to stop: a European perspective. Am J Gastroenterol 2016; 111(8): 1085-1086. DOI: https://doi.org/10.1038/ajg.2016.166

2. Scally B, Emberson JR, Spata E, et al. Effects of gastroprotectant drugs for the prevention and treatment of peptic ulcer disease and its complications: a meta-analysis of randomised trials. Lancet Gastroenterol Hepatol 2018; 3(4): 231-241. DOI: https://doi.org/10.1016/S2468-1253(18)30037-2

3. Khan M, Santana J, Donnellan C, et al. Medical treatments in the short term management of reflux oesophagitis. Cochrane Database Syst Rev 2007; 2: CD003244.

4. Eusebi LH, Rabitti S, Artesiani ML, et al. Proton pump inhibitors: risks of long-term use. J Gastroenterol Hepatol 2017; 32(7): 1295-1302. DOI: https://doi.org/10.1111/jgh.13737

5. Maggio M, Corsonello A, Ceda GP, et al. Proton pump inhibitors and risk of 1-year mortality and rehospitalization in older patients discharged from acute care hospitals. JAMA Intern Med 2013; 173(7): 518-523. DOI: https://doi. org/10.1001/jamainternmed.2013.2851

6. Yang Y-X, Lewis JD, Epstein S, et al. Long-term proton pump inhibitor therapy and risk of hip fracture. JAMA 2006; 296(24): 2947-2953. DOI: https://doi.org/10.1001/jama.296.24.2947

7. Sheen E, Triadafilopoulos G. Adverse effects of long-term proton pump inhibitor therapy. Dig Dis Sci 2011; 56(4): 931-950. DOI: https://doi.org/10.1007/s10620-010-1560-3

8. Medicines and Healthcare Products Regulatory Agency UK. Proton pump inhibitors in long-term use: reports of hypomagnesaemia. 2012; https://www.gov.uk/drug-safety-update/proton-pump-inhibitors-in-long-term-usereports-of-hypomagnesaemia (accessed 6 June 2019)

9. US Food and Drug Administration. FDA Drug Safety Communication: Possible increased risk of fractures of the hip, wrist, and spine with the use of proton pump inhibitors. 2010; https://www.fda.gov/Drugs/DrugSafety/ucm213206. htm\#SafetyAnnouncement (accessed 6 June 2019)

10. Xie Y, Bowe B, Li T, et al. Risk of death among users of proton pump inhibitors: a longitudinal observational cohort study of United States veterans. BMJ Open 2017; 7(6): e015735. DOI: https://doi.org/10.1136/bmjopen-2016015735

11. Jankowski JAZ, de Caestecker J, Love SB, et al. Esomeprazole and aspirin in Barrett's oesophagus (AspECT): a randomised factorial trial. Lancet 2018; 392(10145): 400-408. DOI: https://doi.org/10.1016/S0140-6736(18)313886

12. National Institute for Health and Care Excellence (NICE). Gastro-oesophageal reflux disease and dyspepsia in adults: investigation and management [NICE Clinical Guideline No 184]. 2014; https://www.nice.org.uk/guidance/ cg184 (accessed 6 June 2019)

13. Othman F, Card TR, Crooks CJ. Proton pump inhibitor prescribing patterns in the UK: a primary care database study. Pharmacoepidemiol Drug Saf 2016; 25(9): 1079-1087. DOI: https://doi.org/10.1002/pds.4043

14. UK Health and Social Care Information Centre. Health Survey for England 2016: prescribed medicines. 2017; http:// healthsurvey.hscic.gov.uk/media/63790/HSE2016-pres-med.pdf (accessed 6 June 2019) 
15. Haastrup PF, Paulsen MS, Christensen RD, et al. Medical and non-medical predictors of initiating long-term use of proton pump inhibitors: a nationwide cohort study of first-time users during a 10-year period. Aliment Pharmacol Ther 2016; 44(1): 78-87. DOI: https://doi.org/10.1111/apt.13649

16. Schepisi R, Fusco S, Sganga F, et al. Inappropriate use of proton pump inhibitors in elderly patients discharged from acute care hospitals. J Nutr Health Aging 2016; 20(6): 665-670. DOI: https://doi.org/10.1007/s12603-0150642-5

17. Moriarty F, Bennett $\mathrm{K}$, Cahir $\mathrm{C}$, et al. Characterizing potentially inappropriate prescribing of proton pump inhibitors in older people in primary care in Ireland from 1997 to 2012. J Am Geriatr Soc 2016; 64(12): e291-e296. DOI: https://doi.org/10.1111/jgs.14528

18. De Rijdt T, Spriet I, Willems L, et al. Appropriateness of acid suppression therapy. Ann Pharmacother 2017; 51(2): 125-134. DOI: https://doi.org/10.1177/1060028016670414

19. Batuwitage BT, Kingham JGC, Morgan NE, et al. Inappropriate prescribing of proton pump inhibitors in primary care. Postgrad Med J 2007; 83(975): 66-68. DOI: https://doi.org/10.1136/pgmj.2006.051151

20. Grant K, Al-Adhami N, Tordoff J, et al. Continuation of proton pump inhibitors from hospital to community. Pharm World Sci 2006; 28(4): 189-193. DOI: https://doi.org/10.1007/s11096-006-9028-4

21. Pham CQD, Regal RE, Bostwick TR, et al. Acid suppressive therapy use on an inpatient internal medicine service. Ann Pharmacother 2006; 40(7-8): 1261-1266. DOI: https://doi.org/10.1345/aph.1G703

22. Hungin APS, Hill C, Molloy-Bland M, et al. Systematic review: patterns of proton pump inhibitor use and adherence in gastroesophageal reflux disease. Clin Gastroenterol Hepatol 2012; 10(2): 109-116. DOI: https://doi.org/10.1016/ j.cgh.2011.07.008

23. Labenz J, Labenz G, Stephan D, et al. Insufficient symptom control under long-term treatment with PPI in GERD fact or fiction? MMW Fortschr Med 2016; 158 Suppl 4: 7-11. DOI: https://doi.org/10.1007/s15006-016-8303-0

24. Chey WD, Mody RR, Izat E. Patient and physician satisfaction with proton pump inhibitors (PPIs): are there opportunities for improvement? Dig Dis Sci 2010; 55(12): 3415-3422. DOI: https://doi.org/10.1007/s10620-010$1209-2$

25. Lundell $L$, Vieth $M$, Gibson $F$, et al. Systematic review: the effects of long-term proton pump inhibitor use on serum gastrin levels and gastric histology. Aliment Pharmacol Ther 2015; 42(6): 649-663. DOI: https://doi.org/10.1111/ apt.13324

26. Waldum HL, Hauso $\varnothing$, Fossmark R. The regulation of gastric acid secretion - clinical perspectives. Acta Physiol (Oxf) 2014; 210(2): 239-256. DOI: https://doi.org/10.1111/apha.12208

27. Reimer C, Søndergaard B, Hilsted L, et al. Proton-pump inhibitor therapy induces acid-related symptoms in healthy volunteers after withdrawal of therapy. Gastroenterology 2009; 137(1): 80-87. DOI: https://doi.org/10.1053/j. gastro.2009.03.058

28. Niklasson A, Lindström $L$, Simrén $M$, et al. Dyspeptic symptom development after discontinuation of a proton pump inhibitor: a double-blind placebo-controlled trial. Am J Gastroenterol 2010; 105(7): 1531-1537. DOI: https:// doi.org/10.1038/ajg.2010.81

29. Murie J, Allen J, Simmonds R, et al. Glad you brought it up: a patient-centred programme to reduce proton-pump inhibitor prescribing in general medical practice. Qual Prim Care 2012; 20(2): 141-148.

30. Evans N, Rawaf S, Holmes J, et al. Dyspepsia and GORD care after PPI use. British Journal of Healthcare Management 2007; 13(11): 425-430. DOI: https://doi.org/10.12968/bjhc.2007.13.11.27706

31. Connolly M, Bhatt A, Simmonds R. Implementing UK national dyspepsia guidelines for returning patients to selfcare: findings from an educational intervention and 12-month follow-up clinical re-audit. Gut 2009; 58(Suppl II): A128.

32. Cawston J, Wood N. Implementing a protocol for managing dyspepsia. Prescriber 2003(19): 27-31.

33. Spencer M. Medicines optimisation reviews in patients taking PPIs. Prescriber 2013; 24(9): 20-23. DOI: https://doi. org/10.1002/psb.1051

34. Feinle-Bisset C, Horowitz M. Dietary factors in functional dyspepsia. Neurogastroenterol Motil 2006; 18(8): 608-618. DOI: https://doi.org/10.1111/j.1365-2982.2006.00790.x

35. Sweis R, Kaufman E, Anggiansah A, et al. Post-prandial reflux suppression by a raft-forming alginate (Gaviscon Advance) compared to a simple antacid documented by magnetic resonance imaging and $\mathrm{pH}$-impedance monitoring: mechanistic assessment in healthy volunteers and randomised, controlled, double-blind study in reflux patients. Aliment Pharmacol Ther 2013; 37(11): 1093-1102. DOI: https://doi.org/10.1111/apt.12318

36. Hampson FC, Jolliffe IG, Bakhtyari A, et al. Alginate-antacid combinations: raft formation and gastric retention studies. Drug Dev Ind Pharm 2010; 36(5): 614-623. DOI: https://doi.org/10.3109/03639040903388290

37. Reimer C, Lødrup AB, Smith G, et al. Randomised clinical trial: alginate (Gaviscon Advance) vs. placebo as add-on therapy in reflux patients with inadequate response to a once daily proton pump inhibitor. Aliment Pharmacol Ther 2016; 43(8): 899-909. DOI: https://doi.org/10.1111/apt.13567

38. Strugala V, Dettmar PW, Sarratt K, et al. A randomized, controlled, crossover trial to investigate times to onset of the perception of soothing and cooling by over-the-counter heartburn treatments. J Int Med Res 2010; 38(2): 449-457. DOI: https://doi.org/10.1177/147323001003800208

39. Healthcare RB. Gaviscon Advance - peppermint. Summary of product characteristics. 2014; https://www. medicines.org.uk/emc/product/6715/smpc (accessed 27 Jun 2019)

40. Krol N, Wensing M, Haaijer-Ruskamp F, et al. Patient-directed strategy to reduce prescribing for patients with dyspepsia in general practice: a randomized trial. Aliment Pharmacol Ther 2004; 19(8): 917-922. DOI: https://doi. org/10.1111/j.1365-2036.2004.01928.x

41. Dibley LB, Norton $\mathrm{C}$, Jones R. Non-pharmacological intervention for gastro-oesophageal reflux disease in primary care. Br J Gen Pract 2010; 60(581): e459-e465. DOI: https://doi.org/10.3399/bjgp10X544050 
42. McGinn D, McGinn D, Roberts $C$, et al. Outcomes of a medication optimisation review in patients taking proton pump inhibitors. Guidelines in Practice. 2015; http://d1c7lpjmvlh0qr.cloudfront.net/uploads/i/y/c/McGinn-andRoberts_PPI-supplement.pdf (accessed 27 Jun 2019)

43. Forgacs I, Loganayagam A. Overprescribing proton pump inhibitors. BMJ 2008; 336(7634): 2-3. DOI: https://doi. org/10.1136/bmj.39406.449456.BE

44. NHS Digital. Prescription cost analysis, England [2017]. 2018; https://digital.nhs.uk/data-and-information/ publications/statistical/prescription-cost-analysis/prescription-cost-analysis-england-2017 (accessed 6 June 2019)

45. Theisen J, Nehra D, Citron D, et al. Suppression of gastric acid secretion in patients with gastroesophageal reflux disease results in gastric bacterial overgrowth and deconjugation of bile acids. J Gastrointest Surg 2000; 4(1): 50-54. DOI: https://doi.org/10.1016/S1091-255X(00)80032-3

46. Hussain S, Siddiqui AN, Habib A, et al. Proton pump inhibitors' use and risk of hip fracture: a systematic review and meta-analysis. Rheumatol Int 2018; 38(11): 1999-2014. DOI: https://doi.org/10.1007/s00296-018-4142-x

47. NHS England. Involving people in their own healthy and care: statutory guidance for clinical commissioning groups and NHS England; https://www.england.nhs.uk/wp-content/uploads/2017/04/ppp-involving-people-health-careguidance.pdf (accessed 6 June 2019) 